InnOvaciOnes de NegOciOs 16(31): 143-195

(c) 2019 UANL, Impreso en México (ISSN: 2007-1191)

Recepción: 5 Abril 2018 Aceptación: 22 Abril de 2018

\title{
Factores determinantes del capital de trabajo que influyen en la rentabilidad de las pequeñas empresas en Guayaquil, Ecuador (Determinants of working capital that influence the profitability of small businesses in Guayaquil, Ecuador)
}

\author{
Miriam Rosario Jara Molina, María Margarita Carrera Sánchez, Luis \\ Alberto Villarreal Villarreal, Silverio Tamez Garza \\ Universidad Autónoma de Nuevo León
}

magaly_carrera@hotmail.com, luis.villarrealv@uanl.mx, drsilveriotamez@gmail.com

\begin{abstract}
Nowadays, small businesses in Guayaquil, Ecuador play a fundamental role. In that regard, it is defined the importance of the proper management of the factors of working capital, which contributes significantly to profitability and growth of these companies. This is feasible to achieve through an efficient control and establishing appropriate policies regarding accounts receivable inventories administration, accounts payable and cash conversion cycle, in such a way it collaborates in an effective way in operational decision making of companies. The aim of this preliminary study is to analyze the decisive factors of working capital that influence on profitability of small businesses of Guayaquil, Ecuador. Thus, the main research question is defined: What are the decisive factors of working capital that have an influence on profitability of small businesses of Guayaquil? The results were obtained through the logit method (organized), which led to two significant variables: accounts payable administration and cash conversion cycle on profitability of small businesses.
\end{abstract}

Key words: Accounts receivable, Inventories, Accounts payable, cash conversion cycle, Profitability.

JEL: M41, M42.

Resumen: En la actualidad, el capital de trabajo representa uno de factores centrales de la economía de las Pequeñas Empresas Comerciales (PEC) de la ciudad de Guayaquil en Ecuador. En tal sentido se define la importancia del adecuado manejo de

\section{Factores determinantes del capital de trabajo}


los factores determinantes del capital de trabajo, que contribuyen significativamente en la rentabilidad y crecimiento de estas empresas. Esto es factible de alcanzar llevando un control eficiente y estableciendo políticas adecuadas en la administración de cuentas por cobrar, inventarios, cuentas por pagar y ciclo de conversión de efectivo, de tal manera que, colaboren en forma efectiva en la toma de decisiones operativas de las empresas. El propósito del presente estudio consiste en analizar los factores determinantes del capital de trabajo que influyen en la rentabilidad de las PEC de Guayaquil, Ecuador. Por lo que se define la pregunta central de investigación ¿Cuáles son los factores determinantes del capital de trabajo que influyen en la rentabilidad de las PEC de Guayaquil, Ecuador? Los resultados obtenidos se realizaron a través del método logit (ordenado), lo cual arrojo dos variables significativas: la administración de las cuentas por pagar y el ciclo de conversión del efectivo en la rentabilidad de las PEC.

Palabras claves: Cuentas por cobrar, inventarios, cuentas por pagar, ciclo de conversión del efectivo, rentabilidad.

\title{
Introducción
}

Las PEC en Guayaquil, Ecuador son entes económicos de vital importancia para el crecimiento y desarrollo equilibrado de las actividades productivas del país, ya que son generadoras de fuentes de ingresos y plazas de empleo, el $20 \%$ de la fuerza laboral está empleado en las pequeñas empresas del país. (INEC, 2017) En tal sentido, Shin y Soenen (1998) argumenta que el capital de trabajo es la cantidad de dinero que la empresa necesita día a día para poder mantener el ciclo operativo de la empresa, este ciclo operativo del negocio es el tiempo que transcurre desde que se realiza la compra de las materias primas hasta la cobranza por las ventas realizadas.

\author{
Jara, M., Carrera, M., Villarreal, L., Tamez, S.
}


La eficiente administración de los factores determinantes del capital de trabajo juega un papel muy importante en la rentabilidad en las PEC de Guayaquil, el manejo del capital de trabajo es crucial para el crecimiento y rentabilidad de las pequeñas empresas. (Grablowsky, 1984)

Las pequeñas empresas debido a su tamaño y estructura tienen una serie de limitaciones, ya que no gozan del mismo grado de estabilidad financiera ni de eficiencia operativa que los competidores con mayor capacidad económica y tecnológica.

Por lo tanto, sería imprescindible que los rubros de activos en los que se invierten ciertas cantidades de capital, tales como cuentas por cobrar, inventario, y cuentas por pagar sean bien administradas para de esta manera maximizar la rentabilidad de estas inversiones.

Autores como, Garcia y Martinez (2010) sostienen que la gestión del capital de trabajo afecta a la rentabilidad y riesgo de la empresa y sobre todo sobre a su valor; Perez, Castro, y Garcia (2009), coinciden con el manejo adecuado del capital de trabajo, el cual permitirá a las empresas lograr un crecimiento sostenido y un margen de seguridad para que estás empresas puedan realizar sus actividades operativas de la mejor manera posible, y logren financiarse con capital propio, sin tener que recurrir a una deuda, la cual conlleva a un costo adicional que es el pago de intereses.

El manejo adecuado de los factores determinantes del capital de trabajo les permitirá a estas pequeñas empresas alcanzar un nivel de liquidez óptimo, que le permitirá atender las necesidades que presente el

\section{Factores determinantes del capital de trabajo}


giro del negocio, en todo lo que se refiere a su actividad comercial y afrontar así sus necesidades en el corto plazo.

Las actividades operativas de la empresa son muy variadas y van a estar determinadas por el nivel de ventas, lo cual trae como consecuencia movimientos en el efectivo, en inventario de mercaderías, en cuentas por cobrar (Activos corrientes) y cuentas por pagar (Pasivos corrientes). El capital de trabajo neto se define como la diferencia entre los activos corrientes menos los pasivos corrientes (Gitman, 2012).

Bajo este contexto, el propósito de la presente investigación es probar si se cumple la hipótesis planteada: Si los factores que determinan el capital de trabajo influyen en la rentabilidad de las PEC en Guayaquil, Ecuador. De ahí, el interés de conocer ¿Cuáles son los factores determinantes del capital de trabajo que influyen en la rentabilidad de las PEC de Guayaquil?

Para lograr lo anterior, se efectuó un modelo de tipo Logit (ordenado), en el cual se analizaron los factores determinantes del capital de trabajo, que influyen directa o indirectamente sobre la rentabilidad de las PEC de Guayaquil, Ecuador.

El trabajo está estructurado de la siguiente manera: Se presenta una breve revisión teórica, posteriormente se describe la obtención de los datos y el método de análisis. Enseguida se presentan los resultados y por último algunas conclusiones y recomendaciones.

\section{Marco teórico}

Para analizar la relación entre la gestión del capital de trabajo y la rentabilidad empresarial Deloof (2003), utilizó una muestra de 1,009

Jara, M., Carrera, M., Villarreal, L., Tamez, S. 
grandes empresas no financieras belgas para el período 19921996. Mediante el uso de pruebas de correlación y regresión, encontró resultados negativos significativos entre la relación del ingreso bruto de operación y el número de días de cuentas por cobrar, inventarios y cuentas por pagar de empresas belgas. Con base en los resultados del estudio, sugiere que los gerentes pueden aumentar la rentabilidad de la empresa reduciendo el número de días de cuentas por cobrar y los inventarios, además plantea que el ciclo de conversión de efectivo podría tener efectos positivos y negativos en la rentabilidad de la empresa, por ejemplo, si la empresa tiene un ciclo de conversión de efectivo largo podría tener mayores ventas debido a la larga duración del crédito otorgada es a los clientes, el alto costo de inversión en capital de trabajo podría también disminuir la rentabilidad.

Ghosh y Maji, (2003), examinaron la eficiencia de la gestión del capital circulante de las empresas indias de cemento durante 1992 - 1993 a 2001 - 2002. Mediante el uso de análisis de regresión y las normas de la industria como un nivel de eficiencia objetivo de las empresas individuales, Ghosh y Maji (2003) demostraron que se alcanzó ese nivel objetivo de eficiencia por parte de las empresas durante el período de estudio y encontraron que algunas de las empresas de la muestra tuvieron éxito durante estos años.

Eljelly (2004) examinó empíricamente la relación entre rentabilidad y liquidez, medida por la razón actual y la diferencia de caja es decir el ciclo de conversión de efectivo, en una muestra de 929 sociedades anónimas en Arabia Saudita. Utilizando análisis de correlación y regresión, Eljelly (2004) encontró una relación negativa

\section{Factores determinantes del capital de trabajo}


significativa entre la rentabilidad de la empresa y su nivel de liquidez, medido por la razón actual. Esta relación es más pronunciada para las empresas con altas ratios de corriente y largos ciclos de conversión de efectivo.

Lazaridis y Tryfonidis (2006), realizó un estudio transversal utilizando una muestra de 131 empresas que cotizan en la Bolsa de Atenas para el período 2001-2004 y encontró una relación estadísticamente significativa entre la rentabilidad, medida el beneficio operativo y el ciclo de conversión de efectivo y sus componentes (cuentas por cobrar, cuentas por pagar e inventario). Sobre el análisis de los resultados de los datos anuales mediante pruebas de correlación y regresión, sugieren que los gerentes pueden tener éxito en sus empresas manipulando correctamente el ciclo de conversión de efectivo y manteniendo cada componente del ciclo de conversión (cuentas por cobrar, cuentas por pagar e inventario) en un nivel óptimo.

Padachi (2006) indica que el ciclo de conversión de efectivo es una de las medidas más utilizadas para evaluar y medir los riesgos y beneficios relacionados a la gestión de la liquidez, mide el tiempo que toma en convertir efectivo en efectivo de nuevo, desde el momento en que compra el inventario hasta que este se vende y se cobra por esta venta.

Raheman y Mohamed (2007), estudiaron el efecto de diferentes variables de la gestión del capital de trabajo, el volumen de ventas, el inventario en días, el período medio de pago, el ciclo de conversión de efectivo y el coeficiente de rentabilidad de las empresas paquistaníes. Ellos seleccionaron una muestra de 94 firmas paquistaníes cotizadas en

Jara, M., Carrera, M., Villarreal, L., Tamez, S. 
la Bolsa de Karachi por un período de seis años entre 1999 y 2004 y encontraron una fuerte relación negativa entre las variables de gestión del capital de trabajo y la rentabilidad firma. Encontraron que a medida que aumenta el ciclo de conversión de efectivo, esto conduce a una rentabilidad decreciente de la empresa y los gerentes pueden crear un valor positivo para los accionistas al reducir el ciclo de conversión de efectivo a un posible nivel mínimo.

Garcia y Martinez, (2010), recogió un panel de 8,872 pequeñas y medianas empresas (PYME) procedentes de España que abarcan el período 1996-2002, analizaron los efectos de la gestión del capital circulante sobre la rentabilidad de las PYME utilizando los datos del panel y encontraron una relación negativa entre la rentabilidad y los períodos de cobro y de existencias, concluyendo que los gerentes podrían crear valor reduciendo sus inventarios y el número de días en que sus cuentas están pendientes. Además, la reducción del ciclo de conversión también mejora la rentabilidad de la empresa.

Falope y Ajilore (2009) utilizaron una muestra de 50 empresas no financieras citadas por Nigeria para el período 1996-2005. Su estudio utilizó panel de econometría de datos en una regresión agrupada, donde se combinaron y estimaron series cronológicas y observaciones transversales. Encontraron una relación negativa significativa entre la rentabilidad operativa neta y el período medio de recolección, el inventario volumen de negocios en días, período medio de pago y ciclo de conversión de efectivo para una muestra de cincuenta empresas nigerianas que cotizan en la Bolsa Nigeriana. Además, no encontraron

\section{Factores determinantes del capital de trabajo}


variaciones significativas en los efectos de la gestión del capital circulante entre grandes y pequeñas empresas.

Gill, Nahum, y Neil (2010), investiga la relación entre la gestión del capital de trabajo y la rentabilidad de las empresas para una muestra de 88 empresas de Nueva York por el período de 3 años a partir de 20052007. Encontraron estadísticamente una relación significativa entre el ciclo de conversión de efectivo y la rentabilidad, medida a través del beneficio bruto de explotación. Se deduce que los administradores pueden crear beneficios para sus empresas mediante el manejo correcto del ciclo de conversión de efectivo y mantener las cuentas por cobrar a un nivel óptimo.

La importancia de manejar de manera eficiente el capital de trabajo es innegable para operar con éxito y lograr el crecimiento y sostenibilidad de la empresa ya que provoca efectos sobre la rentabilidad y el riesgo (Tsagem, Aripin, y lshak, 2014).

Tabla 1. Aportaciones Teóricas

\begin{tabular}{cccc}
\hline Autor & Año & País & \multicolumn{1}{c}{ Aportaciones teóricas } \\
\hline $\begin{array}{l}\text { Shin y } \\
\text { Soenen }\end{array}$ & 1998 & California & $\begin{array}{l}\text { Encuentran una relación negativa, el resultado } \\
\text { indica que se puede crear valor para su empresa } \\
\text { reduciendo el ciclo de conversión del efectivo a un } \\
\text { mínimo razonable. }\end{array}$
\end{tabular}

Jara, M., Carrera, M., Villarreal, L., Tamez, S. 
Deloof $2003 \quad$ Bélgica

Ghosh y $2003 \quad$ Indias

Eljelly 2004 Arabia Saudita

Lazaridis y $2006 \quad$ Atenas
Tryfonidis

Brealey,

Myers y 2006

Allen

Baños,

García y

Martínez
El ciclo de conversión de efectivo podría tener efectos positivos y negativos en la rentabilidad de la empresa, por ejemplo, si la empresa tiene un ciclo de conversión de efectivo largo podría tener mayores ventas debido a la larga duración del crédito otorgada al crédito comercial es decir a los clientes, el alto costo de inversión en capital de trabajo podría también disminuir la rentabilidad.

Mediante el uso de análisis de regresión y las normas de la industria como un nivel de eficiencia objetivo de las empresas individuales, Ghosh y Maji probaron que se alcanzó ese nivel objetivo de eficiencia por parte de las empresas durante el período de estudio y encontró que algunas de las empresas de la muestra tuvieron éxito durante estos años.

Encontró una relación negativa significativa entre la rentabilidad de la empresa y su nivel de liquidez, medido por la razón actual. Esta relación es más pronunciada para las empresas con altas ratios de corriente y largos ciclos de conversión de efectivo.

Encontró una relación estadísticamente significativa entre la rentabilidad, medida el beneficio operativo y el ciclo de conversión de efectivo y sus componentes (cuentas por cobrar, cuentas por pagar e inventario).

Los inventarios que se mueven más rápidamente 0 que tengan una mayor rotación tendrán un ciclo de efectivo más corto y por lo tanto generará una mayor rentabilidad.

Encuentran una relación negativa entre la rentabilidad y los períodos de cobro y de existencias, concluyendo que los gerentes podrían crear valor reduciendo sus inventarios y el número de días en que sus cuentas están pendientes. Además, la

\section{Factores determinantes del capital de trabajo}




$\begin{array}{lll}\begin{array}{l}\text { Raheman } \\ \text { y Nasr }\end{array} & 2007 & \text { Pakistan } \\ \text { Falope y } & 2009 & \text { Nigeria } \\ \text { Ajilore } & \end{array}$

Mathuva $2009 \quad$ Nairobi

Gill et al. $2010 \quad \begin{aligned} & \text { Estados } \\ & \text { Unidos }\end{aligned}$ reducción del efectivo o ciclo de conversión también mejora la rentabilidad de la empresa.

Encontraron que a medida que aumenta el ciclo de conversión de efectivo, esto conduce a una rentabilidad decreciente de la empresa y los gerentes pueden crear un valor positivo para los accionistas al reducir el ciclo de conversión de efectivo a un posible nivel mínimo.

No encontraron variaciones significativas en los efectos de la gestión del capital circulante entre grandes y pequeñas empresas.

1. Existe una relación negativa muy significativa entre el tiempo que tarda la empresa en cobrar Clientes (período de cobro de cuentas) y rentabilidad.

2. Existe una relación positiva muy significativa entre el período para convertir los inventarios en ventas (el período de conversión del inventario) y la rentabilidad.

3. Existe un efecto altamente significativo relación positiva entre el tiempo que tarda la empresa en pagar a sus acreedores (plazo medio de pago) y la rentabilidad.

Encontraron estadísticamente una relación significativa entre el ciclo de conversión de efectivo y la rentabilidad, medida a través del beneficio bruto de explotación. Se deduce que los administradores pueden crear beneficios para sus empresas mediante el manejo correcto del ciclo de conversión de efectivo y mantener las cuentas por cobrar a un nivel óptimo.

\section{Jara, M., Carrera, M., Villarreal, L., Tamez, S.}




\begin{tabular}{|c|c|c|c|}
\hline $\begin{array}{l}\text { Tsagem et } \\
\text { al. }\end{array}$ & 2014 & Nigeria & \multirow{2}{*}{$\begin{array}{l}\text { capital de trabajo es innegable para operar con éxito } \\
\text { y lograr el crecimiento y sostenibilidad de la empresa } \\
\text { ya que provoca efectos sobre la rentabilidad y el } \\
\text { riesgo. } \\
\text { Los investigadores explican este resultado negativo } \\
\text { cuando hay un ciclo más corto de la conversión del } \\
\text { efectivo el cual generará más beneficio para una } \\
\text { compañia. Las empresas se consideran que están } \\
\text { bien cuando generan retornos que pueden atraer a } \\
\text { más inversionistas y prestamistas, y así aumentar la } \\
\text { financiación necesaria para el crecimiento o las } \\
\text { necesidades de capital de la empresa. }\end{array}$} \\
\hline $\begin{array}{l}\text { kari y } \\
\text { aidu }\end{array}$ & 2016 & Nigeria & \\
\hline
\end{tabular}

Fuente: Elaboración propia.

\section{Metodología}

¿Cuáles son los factores determinantes del capital de trabajo que influyen en la rentabilidad de las pequeñas empresas del sector comercial de la ciudad de Guayaquil? Para poder dar respuesta a estos datos se toma como unidad de análisis de esta investigación los datos recabados a través de la Superintendencia de Compañías de la ciudad de Guayaquil sobre las pequeñas empresas comerciales que han subido sus estados financieros en la página de dicha institución, en el período 2016.

El análisis se realiza a las pequeñas empresas que tienen personería jurídica, que se encuentran ubicadas en la ciudad de Guayaquil y que se dedican a la actividad comercial, debido a que estas empresas juegan un rol muy importante en la economía del país, por su aporte económico al PIB.

\section{Factores determinantes del capital de trabajo}


Del total de empresas registradas en 2016 , el $90,5 \%$ son microempresas, le sigue la pequeña empresa con el $7,5 \%$ y con ventas anuales entre 100.001 a 1'000.000 con entre 10 y 49 funcionarios, la actividad con mayor número de empresas es el comercio al por mayor y menor, que representan el 36,6\% del total de las empresas (INEC, 2017).

Para la determinación del tamaño de la muestra se utilizó el muestreo aleatorio simple, siendo la población el total de pequeñas empresas comerciales de la ciudad de Guayaquil que han presentado sus Estados Financieros a la Superintendencia de Compañías, dando una muestra de 111 pequeñas empresas, de las cuales se tomó como referencia su número de identificación, su dirección, su tamaño, su actividad, para poder aplicar el instrumento de medición.

El instrumento de medición consistió en una encuesta tipo Likert aplicada a los gerentes, dueños o administradores financieros internos. La escala aplicada para determinar si los factores del capital de trabajo influyen en la rentabilidad de las empresas es del 1 al 5 , donde 5 indica que está totalmente de acuerdo, a 1 que está totalmente en desacuerdo.

El cuestionario fue revisado y validado por expertos en el área financiera, quienes hicieron sus aportaciones para mejorar el instrumento, mejoraron el enfoque de algunas preguntas, dando ideas y sugerencias para que las preguntas tengan una mejor comprensión y por lo tanto las respuestas sean más confiables, además fue validada a través de una prueba piloto que se realizó a un grupo pequeño de pequeñas empresas, lo cual sirvió también para mejorar el instrumento.

La investigación es de tipo descriptivo, el diseño experimental transversal, con enfoque cuantitativo, el trabajo de investigación se

Jara, M., Carrera, M., Villarreal, L., Tamez, S. 
fundamenta con el método científico y la utilización del software econométrico Eviews versión 7 y el SPSS versión 20, para determinar si los factores determinantes del capital de trabajo influyen en la rentabilidad de las PEC de Guayaquil, Ecuador.

Para alcanzar el objetivo planteado en esta investigación que es analizar cómo influyen los factores determinantes del capital de trabajo en la rentabilidad de las PEC de Guayaquil, se efectúa un análisis de tipo Logit (ordenado), lo que permite obtener estimaciones de la probabilidad de que las pequeñas empresas aumenten su rentabilidad, con un adecuado manejo de las variables que son significativas o que influyen en la rentabilidad.

\section{Resultados}

Para obtener la respuesta a esta investigación se seleccionó un método estadístico de análisis logit, empleando el software Ewievs versión 7, en el modelo se han definido las siguientes variables independientes que son: Administración de cuentas por cobrar (X1), administración de inventarios (X2), administración de cuentas por pagar (X3), ciclo de conversión del efectivo (X4), y como variable independiente que la rentabilidad $(\mathrm{Y})$.

Tabla 2. Variables del modelo

\begin{tabular}{cl}
\hline Variables & \multicolumn{1}{c}{ Descripción } \\
\hline X1 & Administración de Cuentas por Cobrar \\
X2 & Administración de Inventarios
\end{tabular}

Factores determinantes del capital de trabajo 
X3 Administración de Cuentas por Pagar

X4 Ciclo de conversión del Efectivo

Y Rentabilidad

Fuente: Elaboración propia

Cabe señalar que se obtuvo un Alfa de Cronbach de 0.854 basado en los 25 ítems considerados en el instrumento de medición en función de las variables independientes y dependiente incluidas en el modelo general, para lo cual se utilizó el paquete estadístico SPSS versión 20 , por ello se concluye a priori que no es necesario eliminar ninguno de los ítems.

Tabla 3: Alfa de Cronbach de todas las variables

\begin{tabular}{cc}
\hline \multicolumn{2}{c}{ Estadísticos de fiabilidad } \\
\hline Alfa de Cronbach & No. De elementos \\
0.854 & 25 \\
\hline
\end{tabular}

Fuente: Elaboración propia basada en el paquete SPSS V20

Si la variable dependiente de un modelo expresa preferencias u opiniones de los individuos sobre un tema determinado en este caso la rentabilidad de las pequeñas empresas comerciales, se utiliza el método de análisis logit ordenado, ya que las alternativas del proceso de decisión expresan implícitamente un orden de utilidad de las distintas observaciones y por lo tanto van a tener un carácter ordinal (Rodriguez y Caceres, 2007).

Con el modelo logit, el resultado que se obtiene es la estimación de la probabilidad de que una variable independiente influya o no sobre

Jara, M., Carrera, M., Villarreal, L., Tamez, S. 
una variable dependiente, también permite identificar cuáles son las variables más importantes o significativas del modelo.

Tabla 4: Evaluación de predicción para especificación ordenada

\begin{tabular}{cccccc}
\hline \multicolumn{5}{c}{ Ecuación estimada } \\
\hline Dep. Valor & Obs. & Correct & Incorrect & $\%$ Correct & \% Incorrect \\
1 & 6 & 1 & 5 & 16.667 & 83.333 \\
2 & 4 & 0 & 4 & 0.000 & 100.000 \\
3 & 12 & 0 & 12 & 0.000 & 100.000 \\
4 & 31 & 1 & 30 & 3.226 & 96.774 \\
5 & 58 & 52 & 6 & 89.655 & 10.345 \\
Total & 111 & 54 & 57 & 48.649 & 51.351 \\
\hline
\end{tabular}

Fuente: Elaboración propia basado en el Eviews V7

Nota: Variable dependiente: RMEDNUEVO, Método Logit ordenado por ML (escala cuadrática)

En datos discrecionales con escala de Likert lo correcto es usar un análisis logit ya que se utiliza una métrica ordenada debido a que se trabaja con escala, esta métrica mide el porcentaje de aciertos del modelo, dando como resultado 54 correctas y 57 incorrectas.

Tabla 5: Correlaciones

\begin{tabular}{ccccc}
\hline & $\begin{array}{c}\text { Administración } \\
\text { de Cuentas por } \\
\text { Cobrar }\end{array}$ & $\begin{array}{c}\text { Administración } \\
\text { de Inventarios }\end{array}$ & $\begin{array}{c}\text { Administración } \\
\text { de Cuentas por } \\
\text { Pagar }\end{array}$ & $\begin{array}{c}\text { Ciclo de } \\
\text { Conversión } \\
\text { de Efectivo }\end{array}$ \\
\hline $\begin{array}{c}\text { Administración } \\
\text { de Cuentas por } \\
\text { Cobrar }\end{array}$ & 1 & 0,157 & 0,230 & 0,387 \\
\hline
\end{tabular}




\begin{tabular}{ccccc}
$\begin{array}{c}\text { Administración } \\
\text { de inventarios }\end{array}$ & 0,157 & 1 & 0,224 & 0,023 \\
$\begin{array}{c}\text { Administración } \\
\text { de Cuentas por } \\
\text { Pagar }\end{array}$ & 0,230 & 0,224 & 1 & 0,231 \\
$\begin{array}{c}\text { Ciclo de } \\
\text { Conversión de } \\
\text { Efectivo }\end{array}$ & 0,387 & 0,023 & 0,231 & 1 \\
\hline
\end{tabular}

Fuente: Elaboración propia basado en el SPSS V-20

Se encontró que no hay relación entre las variables independientes por lo tanto la correlación es muy baja entre las variables independientes.

Tabla 6: Variables Significativas

\begin{tabular}{lllll}
\hline Variable & Coeficiente & Error Std. & z-stadistica & Prob. \\
Cuentas por cobrar & 0.452917 & 0.972361 & 0.465791 & 0.6414 \\
Inventarios & 1.010191 & 0.966004 & 1.045742 & 0.2957 \\
Cuentas por pagar & 1.941581 & 0.980700 & 1.979792 & 0.0477 \\
Ciclo de conversión por pagar & 3.408067 & 1.017765 & 3.348578 & 0.0008 \\
& Puntos de limite & & \\
LIMIT_2:C(5) & 0.452917 & 0.972361 & 0.465791 & 0.6414 \\
LIMIT_3: C(6) & 1.010191 & 0.966004 & 1.045742 & 0.2957 \\
LIMIT_4: C(7) & 1.941581 & 0.980700 & 1.979792 & 0.0477 \\
LIMIT_5:C(8) & 3.408067 & 1.017765 & 3.348578 & 0.0008 \\
\hline
\end{tabular}

Fuente: Elaboración propia basado en Eviews V7

Notas: Muestra: 1 111, Observaciones incluidas I: 111, Número de valor del indicador ordenado: 5, Convergencia lograda de 5 iteraciones, Matriz de covarianza computada utilizando segundas derivadas

Una vez aplicado el modelo Logit (ordenado) se estable que las variables administración de cuentas por pagar y ciclo de conversión del efectivo son significativas, con un nivel de significancia de 0.0057 y

Jara, M., Carrera, M., Villarreal, L., Tamez, S. 
0.0321 respectivamente con un mayor impacto cuentas por pagar, por lo que se requiere hacer impactos marginales.

Tabla 7: Efectos Marginales

\begin{tabular}{cccc}
\hline \multicolumn{4}{c}{ Valores promedio } \\
\hline AMED & BMED & CMED & DMED \\
3,72972973 & 3,7027027 & 4,30630631 & 4,00000000 \\
Incremental & Probabilidad & Categoría & \\
$4 \%$ & $4 \%$ & 1 & \\
$8 \%$ & $4 \%$ & 2 & \\
$18 \%$ & $11 \%$ & 3 & \\
$50 \%$ & $31 \%$ & 4 & \\
$100 \%$ & $50 \%$ & 5 & \\
Total & $100 \%$ & & $50 \%$ \\
\hline \multicolumn{4}{c}{ Máxima probabilidad } \\
\hline
\end{tabular}

Fuente: Elaboración propia.

Los efectos marginales se deben calcular para comprender los efectos parciales de un pequeño cambio en la variable explicativa. Los efectos marginales con los datos medios reflejan que existe un $50 \%$ de probabilidad de que estas empresas mejoren su rentabilidad, así como están, pero si se presenta una mejora en la variable cuentas por pagar y en la variable ciclo de conversión de efectivo podría aumentar la rentabilidad en un 10\%, es decir llegar a $60 \%$.

Discusión

De acuerdo con los resultados obtenidos en la presente investigación sobre la influencia de los factores determinantes del capital de trabajo en

\section{Factores determinantes del capital de trabajo}


la rentabilidad de las PEC de Guayaquil, se obtuvo como resultado que hay 2 variables que son significativas en la rentabilidad de las PEC, esto se debe a que en Ecuador estas pequeñas empresas tienen reducida capacidad económica, por lo que es importante para ellas obtener dinero en efectivo para seguir con su proceso operativo.

En diversos estudios realizados Deloof, (2003), Eljelly, (2004), Garcia y Martinez, (2010), Falope y Ajilore, (2009), y Gill et al. (2010) entre otros, se encuentra una relación negativa entre algunos de los factores del capital de trabajo, ya que a menor días de inventario y menor días de cuentas por cobrar la rentabilidad de la empresa será mayor, en tanto que a mayores días de cuentas por pagar se tendrá mayor liquidez y rentabilidad.

El manejo de las pequeñas empresas es importante en cualquier tamaño de empresa y sobre todo es crucial en las pequeñas empresas de Ecuador, muchas de estas empresas al no tener suficiente capital deben recurrir al sistema financiero, lo cual les resulta complicado de acceder debido a una serie de requisitos que deben cumplir, por lo que basan su esperanza en el cobro de las ventas realizadas y en lograr extender el plazo de pago a los proveedores.

Así que los gerentes inevitablemente tienen que dedicar una cantidad considerable de tiempo y atención todos los días al manejo de sus activos y pasivos corrientes debido a sus formas siempre cambiantes y a la naturaleza del negocio (Miller y Mohan, 1989). La importancia de manejar de manera eficiente el capital de trabajo es innegable para operar con éxito y lograr el crecimiento, rentabilidad y sostenibilidad de la

\author{
Jara, M., Carrera, M., Villarreal, L., Tamez, S.
}


empresa ya que provoca efectos sobre la rentabilidad y el riesgo (Tsagem et al., 2014).

El capital de trabajo es el factor más importante para mantener la liquidez, la supervivencia, la solvencia y la rentabilidad de un negocio y sobre todo el crecimiento (Niresh, 2012).

\section{Conclusiones}

Las variables consideradas en el presente trabajo han pasado las pruebas de fiabilidad del Alfa de Cronbach, dando como resultado dos variables significativas que influyen en la rentabilidad de las PEC de Guayaquil, Ecuador que son las cuentas por pagar y el ciclo de conversión del efectivo.

Las cuentas por pagar son la parte principal de financiamiento a corto plazo para las empresas, ya que este financiamiento no tiene costo de intereses, las empresas financian el capital de trabajo con crédito que les otorgan sus proveedores. Lo importante en estas empresas es lograr maximizar el tiempo que transcurre desde la adquisición de las materias primas hasta el pago de éstas, es decir que el tiempo para pagar a los proveedores sea mucho más amplio que el plazo en el que se cobra a los clientes, sin alterar en ningún momento los antecedentes crediticios de la empresa ante sus proveedores, y lograr mediante estrategias de negociación condiciones que permitan un financiamiento sin costo adicional.

Además es necesario tomar en consideración que si no se maneja correctamente las cuentas por pagar, la empresa se vería obligada a recurrir a un financiamiento en el sistema financiero, lo que

\section{Factores determinantes del capital de trabajo}


implica el pago de intereses y sobre todo conseguir este financiamiento es muy difícil para estas empresas, por los requisitos y garantías que piden estas instituciones, lo cual se les hace muy difícil de cumplir a algunas de ellas y de esta manera no acceden al crédito, por lo que la empresa puede entrar en crisis y hasta llegar a quebrar.

El ciclo de conversión de efectivo es el tiempo que la empresa se va a tardar en recuperar su efectivo por las ventas realizadas, para realizar sus actividades productivas con toda normalidad en el día a día. Si la empresa tiene un ciclo de conversión de efectivo corto permite que la empresa tenga liquidez para pagar sus obligaciones a corto plazo, caso contrario debe recurrir a un financiamiento con el correspondiente pago de intereses, por lo que la empresa debe prestarle especial atención a la administración del efectivo y así evitarse problemas de liquidez.

Como recomendaciones se sugiere a los empresarios llevar una adecuada administración de cada uno de los factores determinantes del capital de trabajo, para que de esta manera su rentabilidad se mantenga o se incremente. Es importante que la empresa procure disminuir los días de las cuentas por cobrar y de los inventarios y aumentar los días de las cuentas por pagar, para que la empresa no tenga problemas de liquidez y no tenga que financiarse con costo adicional.

Cabe recalcar que no solo el capital de trabajo influye en la rentabilidad de las pequenas empresas, hay muchos otros factores que influyen en la rentabilidad de las pequeñas empresas de Guayaquil: como es la competencia, los avances tecnológicos, falta de capital, dificultad para acceder al financiamiento, falta de capacitación en el área financiera de las personas que dirigen la empresa, ya que en la mayoría de las

Jara, M., Carrera, M., Villarreal, L., Tamez, S. 
ocaciones las pequeñas empresas no tienen la facilidad de acceder a estos recursos necesarios para que puedan crecer o sostenerse en el mercado. Por lo que es muy recomendable tomar en cuenta estos factores para futuras investigaciones ya que influyen en la rentabilidad de estas pequeñas empresas.

\section{Referencias}

Baños-Caballero, S., García-Teruel, P. J., \& Martínez-Solano, P. (2016). Financing of working capital requirement, financial flexibility and SME performance. Journal of Business Economics and Management, 17(6), 1189-1204

Crabb, P., Brealey, R. A., Myers, S. C., \& Allen, F. (2006). Solutions manual for Principles of corporate finance. New York: McGraw-Hill//rwin

Deloof, M. (2003). Does working Capital Management affect profitability of Belgian firms? . Journal of Business Finance and Accounting, 573-588

Eljelly, A. M. (2004). Liquidity-profitability tradeoff: an empirical investigation in an emerging market. t. . International Journal of Commerce and Management, 4861

Falope, O. I., \& Ajilore, O. T. (2009). Working capital management and corporate profitability: evidence from panel data analysis of selected quoted companies in Nigeria. Research Journal of Business Management, 73-84

Garcia, P., \& Martinez, P. (2010). Effects of working capital management on SME profitability. International Journal of Managerial Finance, 164-177

Ghosh, S., \& Maji, S. (2003). Working capital management efficiency: a study on the Indian cement industry. The Institute of Cost and Works Accountants of India.

Gill, A., Nahum, B., \& Neil, M. (2010). The Relationship Between Working Capital Management And Profitability: Evidence From The United States. Business and Economics Journal

Gitman, L. (2012). Principios de Administración Financiera Doceava edición. Mexico: Pearson Educación

\section{Factores determinantes del capital de trabajo}


Grablowsky, B. (1984). Financial Management of Inventory. Journal of Small Business Management, Vol 22.

INEC. (2017). Informe de rendición de cuentas período: enero-diciembre 2017. Instituto Nacional de Estadística y Censos. Recuperado de: https://bit.ly/2LUUNZG

Lazaridis, I., \& Tryfonidis, D. (2006). Relationship between working capital management and profitability of listed companies in the Athens stock exchange. Journal of Financial Management and Analysis, 25-26

Mathuva, D. (2009). Capital adequact, cost income ratio and performance of comercial Banks: The Kenyan Scenario. The international journal of applied economics and finance, 3(2). 35-47. doi. 10.3923/ijaef.2009.35.47

Miller, D., \& Mohan, P. (1989). Analysis of profit-linked total factor productivity measurement models at the firm level. Management Science, 35(6). 757-767. Disponible en: https://www.jstor.org/stable/2632120

Niresh, J. (2012). Working capital management \& financial performance of manufacturing sector in Sri Lanka. European journal of business and management, 4(15). 23-30. Disponible en: https://pdfs.semanticscholar.org/9e2a/177cc32987641eb513ecf9e47f8add01 d265.pdf

Padachi, K. (2016). Trendis in working capital management and its impact on firms' performance: An analysis of mauritian small manufacturing firms. International review of business research papers, 2(2). 45-58. Disponible en: http://citeseerx.ist.psu.edu/viewdoc/download?doi=10.1.1.475.2653\&rep=rep 1\&type=pdf

Pérez, J., Castro, O., \& García, B. (2009). Gestión del capital circulante y rentabilidad en PYMES. Revista de contabilidad y dirección, (9). 119-142. Disponible en: https://dialnet.unirioja.es/servlet/articulo?codigo $=3127558$

Raheman, A., \& Nasr, M. (2007). Working capital management and profitability-case of pakistani firms. International review of business research papers, 3(1). 279300.

Disponible

en: https://www.researchgate.net/publication/228727444_Working_capital_mana gement_and_profitability-case_of_Pakistani_Firms

Jara, M., Carrera, M., Villarreal, L., Tamez, S. 
Rodriguez, C., \& Caceres, J. (2007). Modelos de elección discreta y especificaciones ordenadas. Estadística Española, 49(166). 451-471. Disponible en: https://bit.ly/36uTGrl

Shin, H., \& Soenen, L. (1998). Efficiency of working capital management and corporate profitability. Financial practice and education, 8. 37-45.

Tsagem, M., Aripin, N., \& Ishak, R. (2014). Impact of Working Capital Management and Corporate Governance on the Profitability of Small and Medium-Sized Entities in Nigeria. International Journal of Science Commerce and Humanities.

Zakari, M., \& Saidu, S. (2016). The impact of cash conversion cycle on firm profitability: evidence from Nigerian listed telecommunication companies. Journal of Finance and Accounting, 4(6), 342-350. Disponible en: http://article.sciencepublishinggroup.com/html/10.11648.j.jfa.20160406.15.ht $\mathrm{ml}$ 